\title{
Intra-articular Injection of a Dextran Polymer Combined with Antibiotic Medications for Bacterial Infective Arthritis in Dogs: 14 Cases
}

\author{
Matthew A. Lazarus ${ }^{1} \quad$ Stanley E. Kim ${ }^{1} \quad$ Daniel D. Lewis ${ }^{1}{ }^{\circledR} \quad$ Matthew D. Johnson $^{1}$ \\ ${ }^{1}$ Department of Small Animal Clinical Sciences, College of Veterinary \\ Medicine, University of Florida, Gainesville, Florida, United States \\ Address for correspondence Stanley E. Kim, BVSc, MS, Department of \\ Small Animal Clinical Sciences, College of Veterinary Medicine, \\ VCOT Open 2021;4:e104-e110. \\ University of Florida, Gainesville, FL 32610, United States \\ (e-mail: stankim@ufl.edu).
}

\author{
Abstract \\ Keywords \\ - dextran polymer \\ - antibiotics \\ - bacterial infective \\ arthritis \\ - dog
}

Objectives The aim of this study was to describe the use of an intra-articular delivery of a dextran polymer (IADP) for bacterial infective arthritis in dogs.

Materials and Methods Cases of bacterial infective arthritis treated with IADP were reviewed. Resolution of infection was based on joint fluid cytology. Lameness scores, total nucleated cell counts, and per cent neutrophils were monitored throughout treatment.

Results There were 14 dogs included in the study. The stifle was the most commonly affected joint. The median number of injections per dog was 3 with a median of 2 weeks between injections. Amikacin was combined with the dextran polymer in 13 cases and imipenem was used in 1 case. Concurrent oral antimicrobials were used in 12/14 cases, and surgery for infection was performed in 10/14 cases. Cytological evidence of infection resolved in $11 / 14$ cases. Median lameness scores throughout treatment decreased from severe (grade $3 / 4$ ) at initial presentation to mild (grade $1 / 4$ ) at the final recheck. Median total nucleated cell counts and per cent neutrophils decreased from 40,000 white blood cell/ $\mu \mathrm{L}$ and $91 \%$ to 2,200 white blood cell/ $\mu \mathrm{L}$ and $4 \%$ respectively. A transient worsening of lameness was the main side effect documented in 5/14 cases. Conclusion Intra-articular dextran polymer combined with antimicrobials was generally well tolerated; however, the efficacy of IADP was unknown in this population since most dogs received concurrent oral antimicrobials and/or surgery.

\section{Introduction}

Bacterial infective arthritis is a devastating condition in dogs and typically causes severe pain and lameness. ${ }^{1}$ While infection may occur spontaneously and is often associated with chronic osteoarthritis, bacterial infective arthritis is most commonly reported following articular surgery. ${ }^{2}$ The inflammatory processes associated with intra-articular sepsis can invoke destructive alterations of the synovium, bone and cartilage, which can lead to permanent disability. ${ }^{1}$

received

June 16, 2021

accepted after revision

October 4, 2021
DOI https://doi.org/

$10.1055 / \mathrm{s}-0041-1739460$.

ISSN 2625-2325.
Various treatments have been described for bacterial infective arthritis, including systemic and local antimicrobial therapy, joint irrigation, removal of implants and surgical debridement; however, sustained administration of antimicrobials is the essential component of any therapeutic plan. ${ }^{1-4}$ Potential limitations of systemic oral antimicrobial administration, which are most commonly prescribed, ${ }^{5,6}$ include poor owner compliance, ${ }^{7-9}$ low tissue penetration into joints ${ }^{8}$ and systemic side effects. ${ }^{8}$ Sustained intra-articular delivery of antimicrobials is therefore attractive as a

\section{(c) 2021. The Author(s).}

This is an open access article published by Thieme under the terms of the Creative Commons Attribution License, permitting unrestricted use, distribution, and reproduction so long as the original work is properly cited. (https://creativecommons.org/licenses/by/4.0/) Georg Thieme Verlag KG, Rüdigerstraße 14, 70469 Stuttgart, Germany 
potentially more reliable method to obtain high antimicrobial concentrations at the infected site. Polymethyl methacrylate (PMMA) beads, calcium sulphate beads and collagen sponges have all been used for intra-articular antimicrobial delivery. ${ }^{10-13}$ These delivery agents have not garnered much popularity, possibly because surgery is required for implantation, as well as removal when PMMA is used.

A biodegradable dextran polymer matrix (Vetrigel, Royer Animal Health, Frederick, Maryland, United States), originally developed for wound management, can also be used to provide sustained antimicrobial delivery. ${ }^{14-16}$ The product is formed by mixing an oxidized dextran solution with a cross-linking agent and excipients, and has a gel-like consistency. The dextran polymer degrades by hydrolysis in vivo over a 4 to 5 -week period. ${ }^{14}$ In an in vitro study evaluating bioactivity against Staphylococcus aureus, elution of amikacin with clindamycin \pm vancomycin from the dextran polymer occurred rapidly for 24 hours followed by a gradual decline in eluted concentrations over a 10-day period. ${ }^{14}$ Potential advantages of using this carrier for intra-articular antimicrobial delivery include the ability to administer therapeutic agents by percutaneous injection, as well its atraumatic physical characteristics.

There is limited published information regarding the clinical use of the dextran polymer in dogs. In a retrospective study, surgical site infection following tibial plateau levelling osteotomy resolved in all cases when treated with local administration of amikacin and clindamycin impregnated dextran polymer at the explant site prior to closure. ${ }^{15}$ To the authors' knowledge, there have been no studies reporting the use of the intra-articular antimicrobials delivered via the dextran polymer (IADP) in the treatment of bacterial infective arthritis. The purpose of this retrospective study was to report the outcomes and complications of dogs with bacterial infective arthritis treated by IADP.

\section{Materials and Methods}

The medical records for dogs receiving IADP for bacterial infective arthritis between 2015 and 2020 at the University of Florida Small Animal Hospital were reviewed. All owners consented to treatment. Inclusion criteria consisted of presentation with a monoarthropathy, joint fluid cytology with a total nucleated cell count (TNCC) greater than 3,000 cells/ $\mu \mathrm{L}$ and greater than $40 \%$ neutrophils, ${ }^{2}$ and follow-up of at least 1 month following cessation of treatment for the determination of treatment success or failure. Data retrieved from each dog's record included the dogs' signalment, the affected joint, duration of clinical abnormalities, whether or not the dog had prior surgery involving the affected joint, severity of lameness, results of joint fluid cytology and bacterial culture, results of diagnostic imaging of the affected joint, including the presence and severity of osteoarthritis, additional oral antimicrobial administration, type and duration of administration, the number and frequency of treatments with IADP, antimicrobial type delivered with IADP and presence of any comorbidities. The degree of lameness was classified as absent (0), mild (1), moderate (2), severe (toe-touching) (3), or non-weight bearing (4) and recorded for each evaluation.
All intra-articular injections were performed using the same technique under dexmedetomidine sedation (5-15 $\mu \mathrm{g} / \mathrm{kg}$ intravenous). Vital parameters were monitored at least every 5 minutes during the sedation, as well as after sedation until full recovery. Atipamezole $(100-300 \mu \mathrm{g} / \mathrm{kg}$ intramuscular) was used for reversal of sedation in all cases. The affected joint was aseptically prepared. Arthrocentesis was performed and synovial fluid was submitted for cytological analysis; fluid was also submitted for bacterial culture according to feasibility (e.g. sufficient volume) and need (e.g. lack of previous culture). The dextran polymer was prepared according to manufacturer guidelines. ${ }^{17}$ The syringe containing the oxidized dextran solution was combined with a syringe containing the antimicrobial. This syringe was then connected to the syringe containing the cross-linking agent and excipients using the supplied Luer Lock connector. The syringes were mixed by repeat reciprocations for 20 cycles. The antimicrobial selected was based on results of a culture and susceptibility, if available, or empirically chosen by the clinician if susceptibility results were not available. The mixture was then aseptically injected into the affected joint with a $20 \mathrm{~g}$ needle. Repeat injections were recommended every 2 weeks, but the treatment interval could be adjusted according to clinician preference.

The infection was considered to be cytologically resolved if the TNCC was less than 3,000 cells/ $\mu \mathrm{L}$ and if there were less than $40 \%$ neutrophils in joint fluid obtained on follow-up evaluations, as well as improvement without recurrence of lameness for at least 1 month after cessation of treatment. Treatment was considered successful if there was mild or no lameness as documented by the veterinarian and owner, and cytologically resolved according to the definitions above. That is, a case was still classified as having an unsuccessful outcome if there was a persistent moderate-to-severe lameness despite cytologic resolution of infection. Records were also reviewed for potential local complications from the IADP administration such as pain at the injection site, loss of range of motion and worsening of lameness, as well as known systemic adverse effects of the delivered antimicrobial (such as signs of renal injury for aminoglycosides).

\section{Results}

\section{Study Population}

Fourteen dogs met the inclusion criteria ( Table 1). Comorbidities were present in three cases. The stifle was the most commonly affected joint $(n=12)$; there was one elbow and one carpus infection. Eleven stifles had undergone previous surgery. Four joints developed infection without previous surgery. None of the cases had evidence of infection elsewhere. The median duration between the onset of clinical signs of bacterial infective arthritis and presentation was 19 days (range: $2-180$ days).

\section{Lameness Evaluation}

At the time of initial presentation, the median lameness score was 3 (severe lameness) (range: $0-4$ ) (-Fig. 1). The median lameness score decreased at the first recheck to 2 


\begin{tabular}{|c|c|c|c|c|c|c|c|c|c|c|c|c|c|c|}
\hline 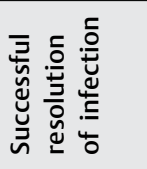 & $\stackrel{\check{\nu}}{\rightleftharpoons}$ & $\stackrel{\check{y}}{\rightleftharpoons}$ & $\stackrel{\check{\nu}}{\nu}$ & 2 & $\stackrel{\check{\varphi}}{\rightleftharpoons}$ & $\stackrel{\check{y}}{\rightleftharpoons}$ & $\stackrel{\check{\varphi}}{\rightleftharpoons}$ & 2o & $\stackrel{y}{\nu}$ & $\stackrel{\check{\varphi}}{\rightleftharpoons}$ & $\stackrel{\check{y}}{\succ}$ & 2 & $\stackrel{y}{\rightleftharpoons}$ & $\stackrel{\check{\Perp}}{\searrow}$ \\
\hline 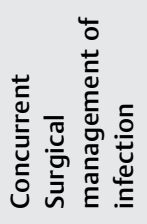 & 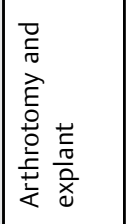 & 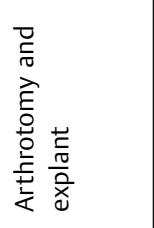 & 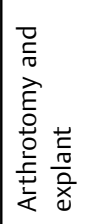 & \begin{tabular}{|l} 
ते \\
总 \\
0 \\
帝 \\
走
\end{tabular} & 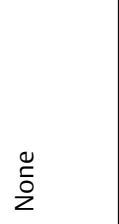 & \begin{tabular}{|l|} 
\\
$\frac{n}{0}$ \\
$\frac{\pi}{2}$ \\
$\dot{x}$
\end{tabular} & 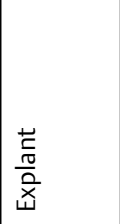 & 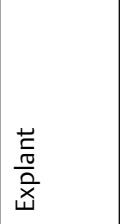 & 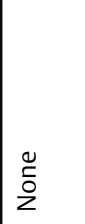 & 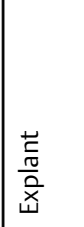 & $\begin{array}{l}0 \\
\tilde{o} \\
\tilde{z}\end{array}$ & 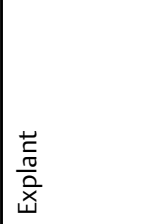 & $\begin{array}{l}0 \\
\tilde{0} \\
\tilde{z}\end{array}$ & 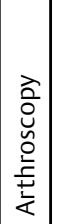 \\
\hline 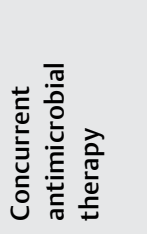 & $\begin{array}{l}0 \\
\check{0} \\
z\end{array}$ & 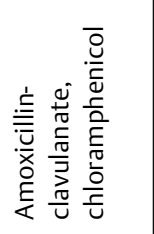 & 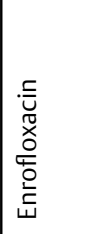 & 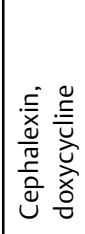 & 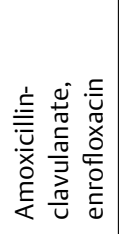 & \begin{tabular}{|l}
0 \\
$\tilde{2}$ \\
$z$
\end{tabular} & 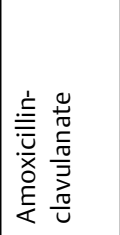 & 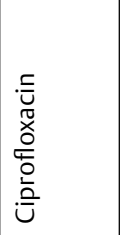 & 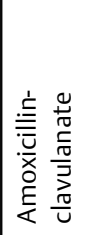 & 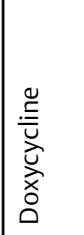 & 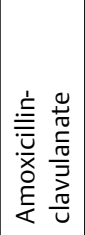 & 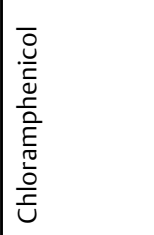 & 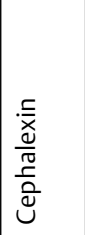 & 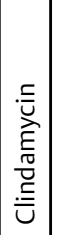 \\
\hline 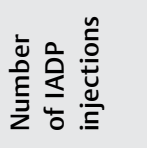 & $\sim$ & - & $m$ & $r$ & in & in & - & $\sim$ & in & $m$ & $m$ & $\sim$ & $\nabla$ & $m$ \\
\hline 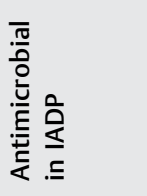 & 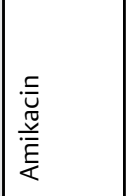 & 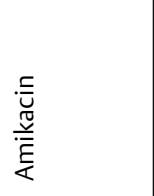 & 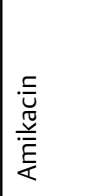 & 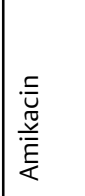 & 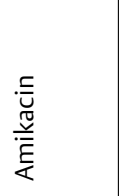 & 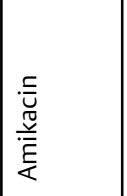 & 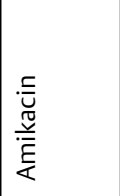 & 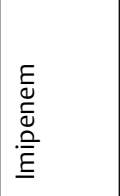 & 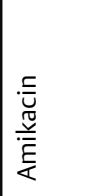 & 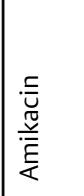 & 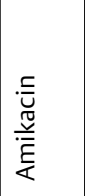 & 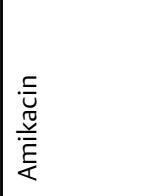 & 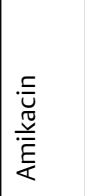 & 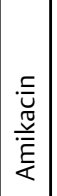 \\
\hline 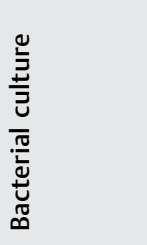 & 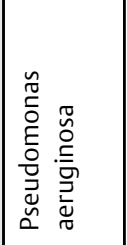 & 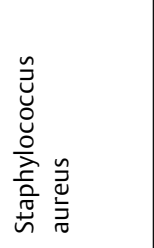 & 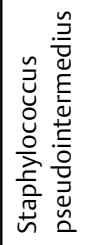 & $\mid \begin{array}{l}5 \\
\frac{1}{3} \\
0 \\
0 \\
0 \\
2\end{array}$ & $\begin{array}{l}\frac{5}{3} \\
3 \\
0 \\
0 \\
2\end{array}$ & \begin{tabular}{|l}
$\frac{1}{1}$ \\
3 \\
0 \\
$\vdots$ \\
0 \\
2
\end{tabular} & $\begin{array}{l}\frac{5}{1} \\
3 \\
0 \\
\vdots \\
0 \\
2\end{array}$ & 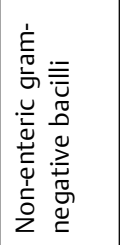 & $\begin{array}{l}5 \\
\text { 音 } \\
0 \\
\vdots \\
0 \\
2\end{array}$ & 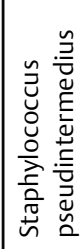 & 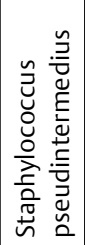 & $\mid \begin{array}{l}\frac{1}{1} \\
3 \\
0 \\
0 \\
0 \\
0 \\
2\end{array}$ & 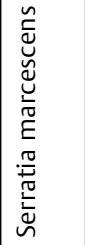 & 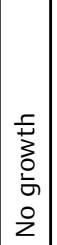 \\
\hline 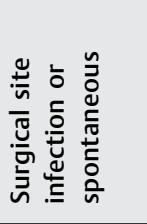 & 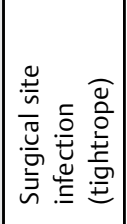 & 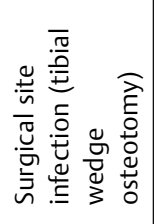 & 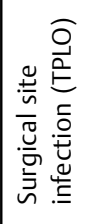 & 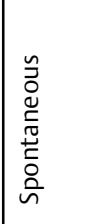 & 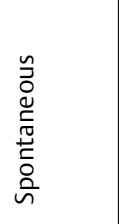 & 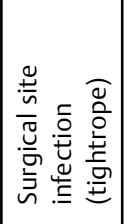 & 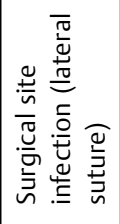 & 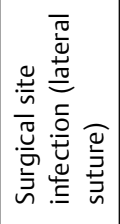 & 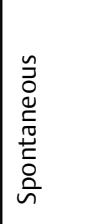 & 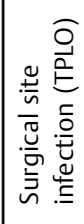 & 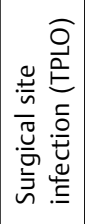 & 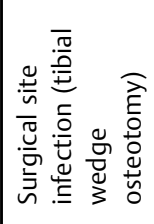 & 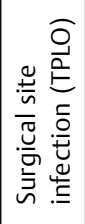 & 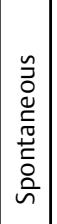 \\
\hline 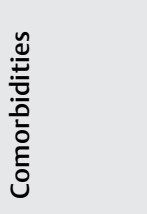 & 蒿 & $\begin{array}{l}\tilde{y} \\
\tilde{\Sigma} \\
z\end{array}$ & 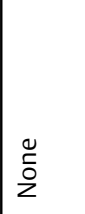 & 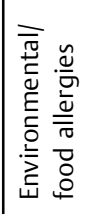 & $\begin{array}{l}\tilde{y} \\
\tilde{\Sigma} \\
z\end{array}$ & $\begin{array}{l}0 \\
\check{0} \\
2\end{array}$ & 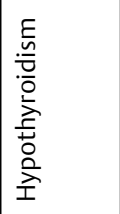 & $\begin{array}{l}0 \\
\tilde{z} \\
\text { ż }\end{array}$ & \begin{tabular}{|l}
0 \\
$\tilde{z}$ \\
$z$
\end{tabular} & $\begin{array}{l}0 \\
\check{0} \\
2\end{array}$ & 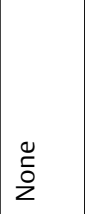 & 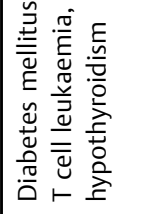 & 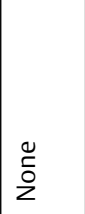 & $\left|\begin{array}{l}0 \\
\vdots \\
z \\
z\end{array}\right|$ \\
\hline 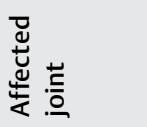 & 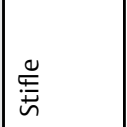 & 尝 & 惡 & \begin{tabular}{|l}
3 \\
0 \\
$\stackrel{0}{w}$
\end{tabular} & 尝 & 惡 & 密 & 臺 & 㴈 & 惡 & 忢 & 惡 & 㴈 & 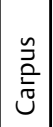 \\
\hline 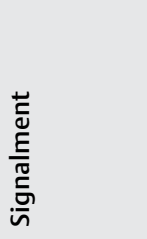 & 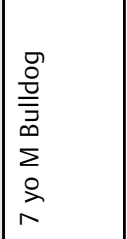 & 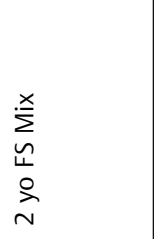 & 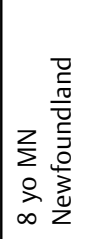 & 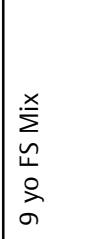 & 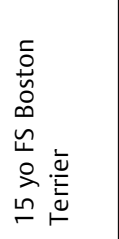 & 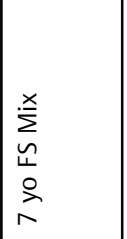 & $\begin{array}{l}\sum_{u}^{x} \\
\tilde{u} \\
0 \\
\infty \\
n\end{array}$ & 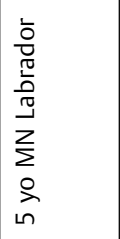 & 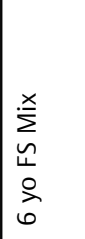 & 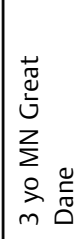 & 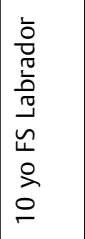 & 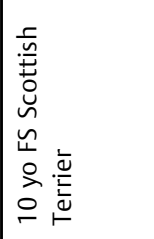 & 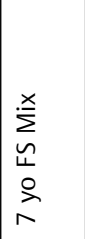 & 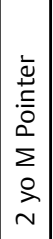 \\
\hline 豹 & - & $\sim$ & $m$ & $\sigma$ & in & 6 & n & $\infty$ & $a$ & $\circ$ & $=$ & $\simeq$ & $\stackrel{m}{n}$ & 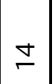 \\
\hline
\end{tabular}




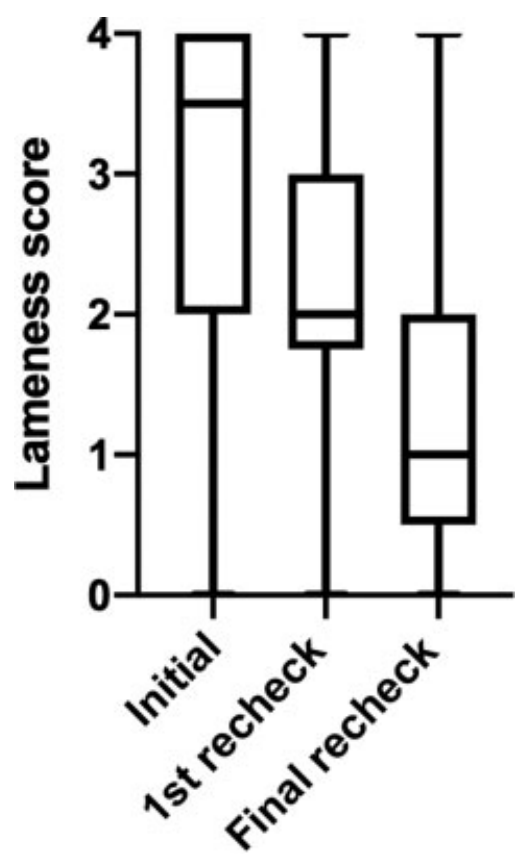

Fig. 1 Box and whiskers plot of lameness scores recorded at the initial presentation, the first recheck exam and the final recheck exam.

(moderate lameness) (range: 0-4) and then further decreased to 1 (mild lameness) (range: $0-4$ ) at the time of the final recheck. Overall, 10/14 cases had improved lameness from the initial evaluation and $4 / 14$ cases did not show signs of improvement, one of which never had a visible lameness. Overall, 11/ 14 dogs had some degree of lameness at the time of the last evaluation.

\section{Cytologic Findings}

The median TNCC and per cent neutrophils on initial cytology were 40,000 white blood cell $(\mathrm{WBC}) / \mu \mathrm{L}$ (range: 3,000-130,000) and 91\% (range: 59-94\%) respectively. Final median TNCC and per cent neutrophils were $2,200 \mathrm{WBC} / \mu \mathrm{L}$ (range: $1,080-30,000)$ and $4 \%$ (range: $1-92 \%$ ) respectively (-Figs. 2 and 3). Overall, the infection cytologically resolved in $11 / 14$ cases, including $3 / 4$ cases with spontaneous infective arthritis and in 8/10 cases with postsurgical infection. Of the three cases that did not resolve, one (case 12) had a synovial fluid TNCC of $30,000 \mathrm{WBC} / \mu \mathrm{L}$ with 92\% neutrophils, and had comorbidities including $\mathrm{T}$ cell leukaemia, diabetes mellitus and hypothyroidism; another case (case 8) had a transient improvement in lameness but persistent neutrophilic inflammation was detected in the synovial fluid (no official cytology report was available for this case at the final evaluation); another case (case 4 ) had a final synovial fluid TNCC of 4,920 WBC/ $\mu \mathrm{L}$ with $32 \%$ neutrophils. This case transferred to another hospital where it was treated with immunosuppressive therapy and died before treatment response to the immunosuppresives could be evaluated.

\section{Radiographic Findings}

Radiographs of the affected joint were performed at the time of initial evaluation in all cases. Osteoarthritis was

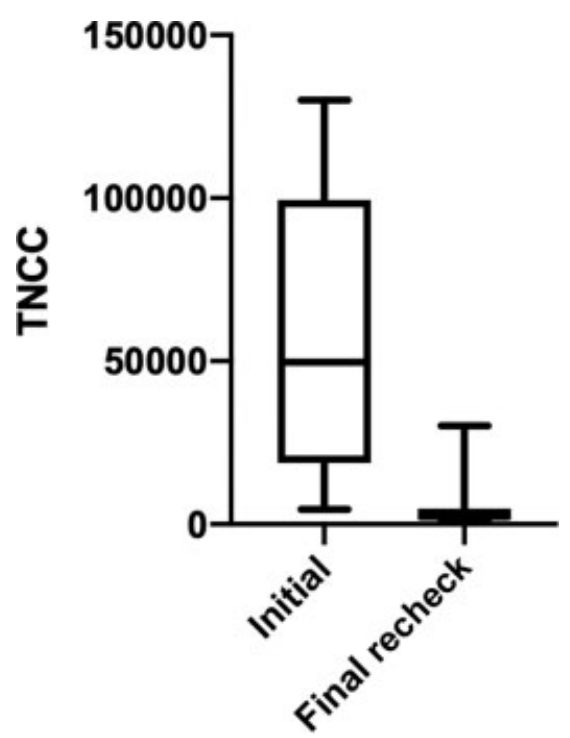

Fig. 2 Box and whiskers plot of initial versus final total synovial fluid nucleated cell counts (TNCC).

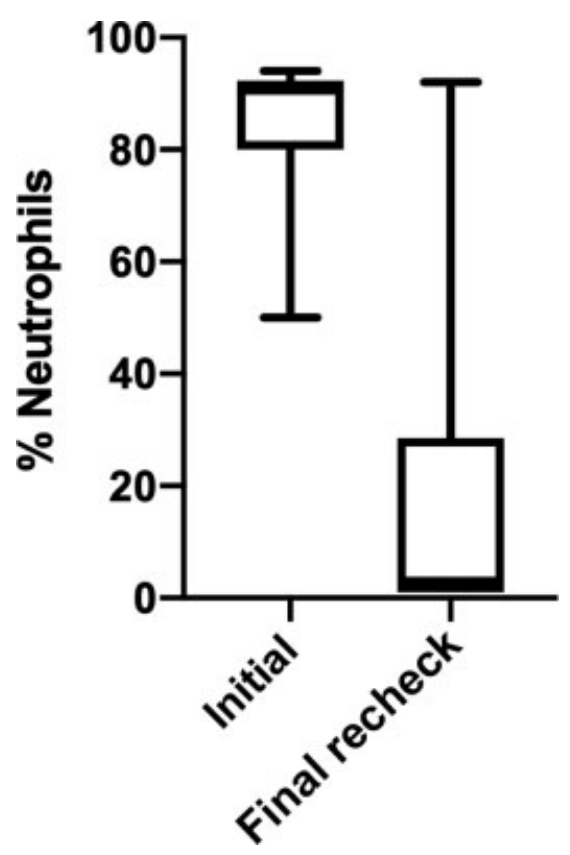

Fig. 3 Box and whiskers plot of initial versus final neutrophil percentage in synovial fluid.

present in all affected joints, and was graded as mild in three cases, moderate in eight cases and severe in three cases. Repeat radiographs were performed in four cases at the end of treatment, all of which showed static osteoarthritic changes when compared with the initial radiographs.

\section{Culture Results}

Bacterial culture of the synovial fluid obtained at the time of or before the first injection was performed in 14 cases of 
which 7 were positive. Organisms cultured included Staphylococcus pseudintermedius $(n=3)$, Staphylococcus aureus $(n=1)$, Pseudomonas aeruginosa $(n=1)$, Serratia marcescens $(n=1)$, and a non-enteric gram-negative bacillus $(n=1)$.

Bacterial culture was performed in three cases after IADP due to lack of cytologic improvement in one case, recurrent lameness and joint effusion in one case, and unspecified reasons in one case. Case 10 had a persistent infection with Staphylococcus pseudintermedius which was initially treated with amikacin in the IADP; the bacteria was deemed susceptible to aminoglycosides; this case eventually had resolution of infection based on cytology and clinical evaluation. The remaining two cases had negative cultures.

\section{IADP Injections}

The median number of dextran polymer injections was 3 per dog (range: 1-7). Thirteen cases were treated with amikacin $(250 \mathrm{mg})$ and one was treated with imipenem $(250 \mathrm{mg})$. The actual volume of the dextran polymer injected was not consistently recorded. The potential maximum median dosage per injection (i.e. if presuming the entire volume was administered) was $9 \mathrm{mg} / \mathrm{kg}$ for both amikacin and imipenem. For cases where culture and susceptibility results were available, the isolated bacteria were susceptible to the antimicrobial used in $4 / 7$ cases. Of the remaining three cases, the antimicrobial chosen did not have an interpretation on the susceptibility panels. The infection was resolved cytologically in $6 / 7$ cases with a positive initial culture. The bacteria isolated were sensitive to the antimicrobial combined with the IADP in 4/7 cases and the infection was cleared in $3 / 4$ of those cases. Imipenem was used in the one infection that did not resolve despite the bacteria being sensitive to the antimicrobial. Of the three cases where the bacteria was not deemed sensitive to the chosen antimicrobial, one was treated with amikacin but amikacin was not listed on the susceptibility panel, and there was no interpretation for the injected antimicrobial for the other two cases. When more than one injection was given, treatment frequency ranged from 1 to 4 weeks (median: 2 weeks) between injections. No dogs were deemed to have acute worsening of lameness upon recovery from sedation; however, owners reported transient worsening of lameness in 5/14 cases lasting for 1 to 3 days after the injection. No adverse systemic reactions were noted.

\section{Systemic Antimicrobials}

Twelve total cases received systemic antimicrobial therapy concurrently with IADP including amoxicillin/clavulanic acid $(n=3)$, enrofloxacin $(n=3)$, chloramphenicol $(n=3)$, doxycycline $(n=2)$, ciprofloxacin $(n=1)$, cephalexin $(n=1)$, and clindamycin $(n=1)$. Three dogs were treated with multiple systemic antimicrobials including enrofloxacin with chloramphenicol, cephalexin with doxycycline and amoxicillin/clavulanic acid with enrofloxacin. There were two cases that were treated with the IADP alone and no additional systemic antimicrobial therapy. Both of these cases also received surgery for implant removal. The infection resolved cytologically in both of these cases.

\section{Surgery}

Ten joints were treated surgically within 1 month before the first injection, including implant removal $(n=8)$, carpal arthroscopy $(n=1)$ and lavage of the elbow joint via arthrotomy at the time of biopsy $(n=1)$.

\section{Overall Clinical Outcome}

Treatment was determined to be a success in 11/14 cases, as evidence of infection was resolved cytologically and lameness was noted as improved by both owner and veterinarian.

\section{Discussion}

Cytologic evidence of bacterial infective arthritis resolved in the majority of dogs (11/14) treated with IADP. While our results are promising, we cannot definitively ascribe the positive response to IADP alone since most dogs received concurrent oral antimicrobials, and many underwent surgical debridement. The intra-articular injections were generally well tolerated.

The resolution rate in our cases was similar to other clinical reports describing treatment of bacterial infective arthritis in small dogs, which ranged from 79 to $100 \%$. ${ }^{2,3,18,19}$ Comparing our rates of cytologic resolution of infection to previous reports is difficult for several reasons including heterogenous and varying aetiologies, affected regions and chronicity. In particular, it is possible that our cases encountered greater antimicrobial resistance, as most of our cases had surgical site infection and were therefore likely exposed to prior antimicrobial therapy. ${ }^{20,21}$ Subject numbers were low, and the outcomes reported here and in previous studies may not be reflective of a broader population.

Residual lameness despite resolution of infection was a common finding in our cases. The residual lameness may have been due to many factors including irreversible pathology from the septic process, pre-existing osteoarthritis, progression of osteoarthritis or even a low-grade infection that was not detectable. Eleven of the 14 dogs in this study had some degree of lameness at the time of the last evaluation and all dogs in the study had evidence of osteoarthritis on radiographs at the time of the initial evaluation. Most dogs were only mildly lame at final follow-up, which was consistent with previous studies. ${ }^{2,3,18}$

A positive bacterial culture of synovial fluid was obtained in only $7 / 14$ cases. Bacterial synovial fluid culture is a poorly sensitive test, and limited positive culture rates (44-81\%) were previously reported for small animals with bacterial infective arthritis. ${ }^{1,2,18,22,23}$ We identified cases with negative cultures responding to antibiotic medication therapy as evidenced by improvements in lameness and synovial fluid cytology. Rarely, immune-mediated arthropathy can occur in a single joint, ${ }^{5}$ and it is possible that one of the treatment failures in our report (which had negative cultures) was misdiagnosed and had an autoimmune process. Our overall results, however, corroborate previous evidence demonstrating that a negative synovial fluid culture alone does not exclude bacterial infective arthritis as a differential diagnosis. ${ }^{2,18,22}$ 
Response to treatment can be influenced by factors such as antimicrobial choice, chronicity of infection and comorbidities. Antimicrobial therapy was based on culture and susceptibility results when available. When susceptibility panels were not available, antimicrobial selection was based on clinician experience. No susceptibility profiles were available for $2 / 3$ cases in which the infection did not resolve, leaving the possibility of inappropriate antimicrobial selection as a cause of failure in those cases. It was not possible to determine the influence of infection chronicity in our case series due to low subject numbers. One case with persistent infection may have been immunocompromised as it was diagnosed with several comorbidities including diabetes mellitus, T cell leukaemia and hypothyroidism. Overall, there were no clear associations or patterns for treatment failure in our case series.

The recommended interval for IADP of every 2 weeks was empirical based on a balance between reported elution rates and logistical considerations. ${ }^{14,16}$ There are no established guidelines for treatment frequency using the dextran polymer. In an in vitro study, rapid release of amikacin was observed in the first 24 hours, and fell below breakpoint minimum inhibitory concentration by day $10 .^{14}$ To the authors' knowledge, elution characteristics of antimicrobials from this dextran polymer have only been investigated in vivo in the subcutaneous space of horses, where amikacin was also reported to drop below minimum inhibitory concentrations by day $10 .^{16}$ As no studies have been performed assessing elution or degradation rates within joints, whether appropriate antimicrobial delivery was achieved in our cases remains unknown. We did not detect any evidence that the dextran polymer was still present within joints at subsequent injections. We were concerned about client compliance if repeat injections, which require sedation, were recommended at more frequent (such as weekly) intervals. The ideal regimen needs further investigation, and we caution against using the results or protocols in our study as supporting evidence for a 2-week treatment interval.

No major adverse events were observed with IADP. The potential maximum median dose of antimicrobial in each injection was $9 \mathrm{mg} / \mathrm{kg}$, which was within or below the recommended daily systemic doses of amikacin (15-30 $\mathrm{mg} / \mathrm{kg}$ every 24 hours) and imipenem $(5-10 \mathrm{mg} / \mathrm{kg}$ every 6-8 hours). ${ }^{24}$ In the in vivo equine study, systemic levels of amikacin were not detected at 24 hours and 7 days. ${ }^{16}$ It is therefore unsurprising that known adverse systemic effects of amikacin such as nephrotoxicity were not encountered. Furthermore, we did not combine amikacin with other potentially nephrotoxic or incompatible antimicrobials. Routine monitoring for renal injury (such as identification of renal casts) was not performed; however, based on the doses administered, they were not indicated. Worsening of lameness following IADP in some cases was transient, and may have been caused by distension of the joint rather than any chemically irritating effects of the IADP.

The retrospective design is a major limitation of this study. The number of cases was low, and the population was heterogenous. Definitive resolution of infection was not clinically feasible due to lack of cultures at the end of treatment, and lack of long-term follow-up. As discussed, concurrent treatment methods between cases varied and were not standardized, and it was not possible to determine the efficacy of IADP in this population. It was not surprising to find that most dogs received concurrent oral antimicrobial therapy, given that the efficacy of IADP was (and still is) unknown. While attempts were made to maintain a consistent protocol for IADP, the number and frequency of dosing were variable. These limitations may be difficult to overcome in future studies, since bacterial infective arthritis is uncommon and clinical decision making for these challenging cases is multifactorial.

Summarily, IADP combined with systemic antimicrobial therapy and/or surgery was successful in treating bacterial infective arthritis in the majority of cases. Improvement in lameness scores and cytologic characteristics of synovial fluid were observed. The injections seemed to be well tolerated by all dogs with a minority showing a transient increase in the degree of lameness. Future studies should aim to determine antimicrobial elution and degradation rates within joints, and report outcomes of IADP treated dogs without concurrent antimicrobial therapy.

\section{Conflict of Interest}

The authors declare no conflict of interest.

Acknowledgments

There was no proprietary interest or funding provided for this project.

\section{References}

1 Bennett D, Taylor DJ. Bacterial infective arthritis in the dog. J Small Anim Pract 1988;29:207-230

2 Clements DN, Owen MR, Mosley JR, Carmichael S, Taylor DJ, Bennett D. Retrospective study of bacterial infective arthritis in 31 dogs. J Small Anim Pract 2005;46(04):171-176

3 Benzioni H, Shahar R, Yudelevitch S, Milgram J. Bacterial infective arthritis of the coxofemoral joint in dogs with hip dysplasia. Vet Comp Orthop Traumatol 2008;21(03):262-266

4 Diefenbeck M, Mückley T, Hofmann GO. Prophylaxis and treatment of implant-related infections by local application of antibiotics. Injury 2006;37(Suppl 2):S95-S104

5 Innes JF. Arthritis. In: Johnston SA, Tobias KM, eds. Veterinary Surgery Small Animal. St. Louis: Elsevier; 2018:1265-1299

6 Hayes G, Moens N, Gibson T. A review of local antibiotic implants and applications to veterinary orthopaedic surgery. Vet Comp Orthop Traumatol 2013;26(04):251-259

7 Adams VJ, Campbell JR, Waldner CL, Dowling PM, Shmon CL. Evaluation of client compliance with short-term administration of antimicrobials to dogs. J Am Vet Med Assoc 2005;226(04): 567-574

8 Boothe DM. Interpreting culture and susceptibility data in critical care: perks and pitfalls. J Vet Emerg Crit Care (San Antonio) 2010; 20(01):110-131

9 Van Vlaenderen I, Nautrup BP, Gasper SM. Estimation of the clinical and economic consequences of non-compliance with antimicrobial treatment of canine skin infections. Prev Vet Med 2011;99(2-4):201-210 
10 Buchholz HW, Elson RA, Engelbrecht E, Lodenkämper H, Röttger J, Siegel A. Management of deep infection of total hip replacement. J Bone Joint Surg Br 1981;63-B(03):342-353

11 Ham K, Griffon D, Seddighi M, Johnson AL. Clinical application of tobramycin-impregnated calcium sulfate beads in six dogs (20022004). J Am Anim Hosp Assoc 2008;44(06):320-326

12 Hayes G, Gibson T, Moens NM, et al. Intra-articular implantation of gentamicin impregnated collagen sponge causes joint inflammation and impaired renal function in dogs. Vet Comp Orthop Traumatol 2016;29(02):159-163

13 Owen MR, Moores AP, Coe RJ. Management of MRSA septic arthritis in a dog using a gentamicin-impregnated collagen sponge. J Small Anim Pract 2004;45(12):609-612

14 Thomas LA, Bizikova T, Minihan AC. In vitro elution and antibacterial activity of clindamycin, amikacin, and vancomycin from Rgel polymer. Vet Surg 2011;40(06):774-780

15 Reed TP, Thomas LA, Weeren FR, Ruth JD, Anders BB. A novel dextran polymer hydrogel local antimicrobial therapy in dogs: a pilot study. Can Vet J 2016;57(02):189-195

16 Hart SK, Barrett JG, Brown JA, Papich MG, Powers BE, Sullins KE. Elution of antimicrobials from a cross-linked dextran gel: in vivo quantification. Equine Vet J 2013;45(02):148-153
17 Royer Biomedical Inc. 2019. Royer biomedical. Accessed October 24, 2021, Retrieved from http://www.royerbiomedical.com

18 Marchevsky AM, Read RA. Bacterial septic arthritis in 19 dogs. Aust Vet J 1999;77(04):233-237

19 Mielke B, Comerford E, English K, Meeson R. Spontaneous septic arthritis of canine elbows: twenty-one cases. Vet Comp Orthop Traumatol 2018;31(06):488-493

20 Tam VH, Louie A, Fritsche TR, et al. Impact of drug-exposure intensity and duration of therapy on the emergence of Staphylococcus aureus resistance to a quinolone antimicrobial. J Infect Dis 2007;195(12):1818-1827

21 Marra AR, de Almeida SM, Correa L, et al. The effect of limiting antimicrobial therapy duration on antimicrobial resistance in the critical care setting. Am J Infect Control 2009;37(03): 204-209

22 Scharf VF, Lewis ST, Wellehan JF, et al. Retrospective evaluation of the efficacy of isolating bacteria from synovial fluid in dogs with suspected septic arthritis. Aust Vet J 2015;93(06):200-203

23 Soontornvipart K, Kohout P, Proks P. Septic arthritis in dogs: a retrospective study of 20 cases. Acta Vet Brno 2003;72:405-413

24 Baggot JD, Ling GV, Chatfield RC. Clinical pharmacokinetics of amikacin in dogs. Am J Vet Res 1985;46(08):1793-1796 